\title{
Complications of Endomyocardial Biopsy in Heart Transplant Patients: A Retrospective Study of 2117 Consecutive Procedures
}

\author{
F. Saraiva, V. Matos, L. Gonçalves, M. Antunes, and L.A. Providência
}

\begin{abstract}
Background. Endomyocardial biopsy (EMB) remains the gold standard for the diagnosis of graft rejection after heart transplantation (HT). Our purpose was to evaluate the rate of complications of this invasive procedure.

Methods. This was a retrospective study of 175 patients, who were transplanted between November 2003 and October 2010 and survived more than 1 month after surgery. We evaluated the number of inconclusive EMB and described the incidence, nature, and subsequent management of several complications associated with this procedure.

Results. Over a period of approximately 7 years, we performed 2217 EMB yielding 4972 specimens, namely, an average of 2.3 fragments per procedure. The majority of EMBs $(95.3 \%)$ were performed by the femoral approach. Only 12 EMB $(0.57 \%)$ were inconclusive. The overall complication rate was $0.71 \%$. During puncture, one patient experienced a vasovagal reaction and another one, a femoral artery false aneurysm. During the biopsy, there was one case of cardiac perforation with tamponade, two cases of supraventricular tachycardia, and three atrioventricular conduction abnormalities. In 19 patients, histological analysis revealed chordal tissue, but only two patients developed mild tricuspid regurgitation. We observed five cases of coronary artery fistulae. The clinical outcomes were favorable in all cases.
\end{abstract}

Conclusion. EMB proved to be a suitable, safe method to monitor rejection after HT.

$\mathbf{H}$ EART FAILURE (HF) remains a major cause of mortality, occurring within first 8 years after diagnosis among $80 \%$ of men and $70 \%$ of women under the age of 65 years. ${ }^{1}$ Indeed, despite therapeutic innovations, the morbidity and mortality of advanced HF patients remain high. Therefore, when functional capacity is severely impaired, despite optimal medical therapy, orthotopic heart transplantation (OHT) appears, in most cases, to be the only option. In the United States, it is estimated that about 2000 patients with advanced HF are transplanted each year. $^{2}$ Restrictive selection criteria for donors and recipients, deeper understanding of immune system physiology, development of new immunosuppressive drugs, and better surgical technique have improved the results. The experience of the Mayo Clinic reported above 90\% 1-year survival after OHT and 5-year survival near $80 \% .^{2}$ The improved outcomes of heart transplant patients over the past 30 years is also a consequence of an increased ability to diagnose rejection. In fact, the myocardial histological analysis re- veals several pathological findings that precede clinical manifestations of allograft rejection, allowing an early diagnosis.

Endomyocardial biopsy (EMB) is an invasive procedure, performed first by Sutton et al in 1956, using a transthoracic needle approach. ${ }^{3}$ In 1962, Sakakibara and Konno performed a transvenous EMB using the Konno bioptome. ${ }^{4}$ Later, in 1974, Caves and colleagues developed a technique and instrumentation for percutaneous access, through the internal jugular vein, ${ }^{5}$ allowing the procedure to be per-

From the Department of Cardiology of the Coimbra Hospital and Medical School (F.S., V.M., L.G., L.A.P.), and Department of Cardiothoracic Surgery of the Coimbra Hospital and Medical School (M.A.), Coimbra Hospital and Medical School, Coimbra, Portugal.

Address correspondence to Fátima Saraiva, MD, Department of Cardiology, Coimbra Hospital and Medical School, Praceta Dr. Mota Pinto, 3004 - 561 Coimbra, Portugal. E-mail: saraiva. fatima@gmail.com

(C) 2011 by Elsevier Inc. All rights reserved. 360 Park Avenue South, New York, NY 10010-1710 
formed with only local anesthesia. In 1984, the femoral vein was described as an alternative vascular access. ${ }^{6}$ At present, EMB is a routine procedure, remaining the gold standard to monitor rejection among cardiac transplant patients. However, despite its growing applicability, the procedure is not free of complications and limitations. This study sought to define the safety of this invasive procedure through the description and quantification of its complications.

\section{PATIENTS AND METHODS}

We included all 175 patients who underwent OHT from November 2003 through October 2010 and survived more than 1 month at a single heart transplantation center. The follow-up varied from 1 month to approximately 7 years $($ mean $=3.2 \pm 2.0$ ).

Two interventional cardiologists performed 2.117 EMB according to the following schedule: weekly for the first month, every 2 weeks for the second month, every 3 weeks for the next 4 months, and every 2 months thereafter until the twelfth month. In addition to EMB, patients also underwent right and left heart catheterization and coronary angiography at 1,3 , and 5 years after OHT. Another EMB was performed at 1.5 years. Additional biopsies were obtained when there was a significant suspicion of rejection despite adjustment of immunosuppressive therapy.

Procedures were performed without premedication. The femoral and internal jugular veins were the only vascular access. The puncture was performed under local anesthesia (1\% lidocaine). Using the Seldinger technique, we introduced a sheath (7French/90 cm). A Cordis bioptome (7-French/104 cm) was then advanced slowly, through the sheath, to the apical segment of the right side of the interventricular septum (IVS), using fluoroscopic guidance. Myocardial specimens were sent to the pathology department in $10 \%$ formalin.

All patients were continuously monitored during and after EMB. A 12-lead electrocardiogram and a transthoracic echocardiogram were performed before and after all the EMB procedures, respectively.

The following demographic and patient characteristics were analyzed: age, gender, etiology of HF, number of EMB procedures and collected fragments, vascular access route, as well as type and rate of complications, including death, cardiac perforation requiring urgent pericardiocentesis or cardiac surgery, pneumothorax and hemothorax, embolic stroke, arrhythmias, tricuspid regurgitation (TR), or coronary artery fistula (CAF). We also reported complications during the venous puncture.

\section{RESULTS}

We performed a OHT for end-stage congestive HF in 175 patients aged 4 to 72 years $($ mean $=52.4 \pm 12.7 \mathrm{y})$. The majority were men $(n=135 ; 77.1 \%)$. Dilated cardiomyopathy, the most prevalent clinical diagnosis, was identified in approximately half of the population $(n=82,46.8 \%)$, and ischemic cardiomyopathy in one-third of cases $(n=58$; $33.1 \%$ ).

Over approximately 7 years, we performed 2117 EMB procedures. We preferentially used the femoral approach ( $n=2018$; 95.3\%); only $99 \mathrm{EMB}$ were performed through the right internal jugular vein. Among 4972 harvested fragments $(2-7$ per procedure; mean $=2.3), 4 \%$ did not correspond to myocardial tissue. The majority of the inad- equate fragments were fibrous tissue, probably from a previous biopsy scar. We also observed samples of valvular tissue, fat, and thrombi. Among all of the EMB, only 12 were considered inconclusive $(0.57 \%), 89 \%$ revealed no rejection but approximately $9 \%$ showed mild, $1.3 \%$ moderate, and three severe rejection episodes.

The cumulative incidence of complications was $0.71 \%$ (Table 1). During the puncture, a moderate vasovagal reaction was documented in one patient who became pale, sweaty, and hypotensive. After fluid administration, he experienced total recovery. Another patient developed a false aneurysm of the right common femoral artery after the seventh EMB, as confirmed by echo Doppler. Percutaneous closure was achieved using intra-arterial injection of collagen and thrombin.

We did not identify any case of death related to the EMB procedure. Only one patient had a cardiac perforation during the third EMB. He complained of sudden chest pain, dyspnea, and severe hypotension. A transthoracic echocardiogram confirmed the presence of a pericardial effusion, provoking right diastolic collapse. Surgical drainage was immediately performed to stabilize the patient. There were no recorded cases of hemothorax, pneumothorax, or embolic stroke.

Relatively to arrhythmias, we identified a single case of atrial fibrillation, which developed during the 15th EMB. The patient remained asymptomatic with stable vital signs, spontaneously converting to sinus rhythm, within 24 hours. Another patient developed atrial flutter with a rapid ventricular rate (150 beats per minutes) during the first EMB. Although he developed no symptoms or hemodynamic instability, a successful electrical cardioversion was performed after 12 hours. Abnormalities of atrioventricular (AV) conduction were documented in three patients. Two cases developed transient second-degree block with 2:1 conduction. The third subject showed transitory complete AV block, during bioptome positioning. He previously

Table 1. Complications Associated with 2117 Endomyocardial Biopsies

\begin{tabular}{lll}
\hline & $n$ & $\%$ \\
\hline Death & 0 & 0 \\
Cardiac perforation & 1 & 0.05 \\
Pneumothorax & 0 & 0 \\
Hemothorax & 0 & 0 \\
Embolic stroke & 0 & 0 \\
Arrhythmias & & \\
$\quad$ Supraventricular tachycardia & 2 & 0.09 \\
$\quad$ Ventricular tachycardia & 0 & 0 \\
Conduction abnormalities & & 0.05 \\
$\quad$ Complete heart block & 1 & 0.09 \\
$\quad$ Second degree AV block & 2 & 0.05 \\
False aneurysm & 1 & 0.05 \\
Vasovagal reaction & 1 & 2.8 \\
Coronary artery fistula & 5 & 1.1 \\
Tricuspid regurgitation & 2 & \\
\hline$\quad$ Av, atrioventricular. & &
\end{tabular}

$\mathrm{Av}$, atrioventricular. 
displayed left bundle branch block. None of the three patients required therapeutic intervention.

Valvular tissue was identified during the histological analysis in 19 transplant patients (10.8\%). Subsequent echocardiograms showed no development or worsening of TR in the majority of cases. Only two patients developed mild TR without increased pulmonary artery systolic pressure.

CAF was identified in five patients $(2.8 \%$; Fig 1 and Fig 2). Except for one case, all were detected during coronary angiography, performed routinely at 1 year after OHT. All fistulae ended in the right ventricle and were small in size and asymtomatic. A conservative approach was adopted in all cases. After 2 years, one fistula maintained the same angiographic appearance and another had completely resolved. The remaining three cases have not yet undergone angiography for further comparative study. The histological analysis of the fragments, collected prior to the detection of the CAF, did not reveal the presence of dilated arterioles.

\section{DISCUSSION}

This survey describes complications of EMB. In contrast to some groups, we preferably use the femoral vein for vascular access. Previously published data have already demonstrated that this approach allows an effective appropriate EMB in a greater percentage of cases compared to the jugular access. ${ }^{7}$ Furthermore, the jugular vein is also associated with a higher rate of complications, such as pneumothorax, hemothorax, carotid artery injury, and paralysis of the recurrent laryngeal nerve. ${ }^{8}$ However, it is associated with greater patient discomfort particularly dur-

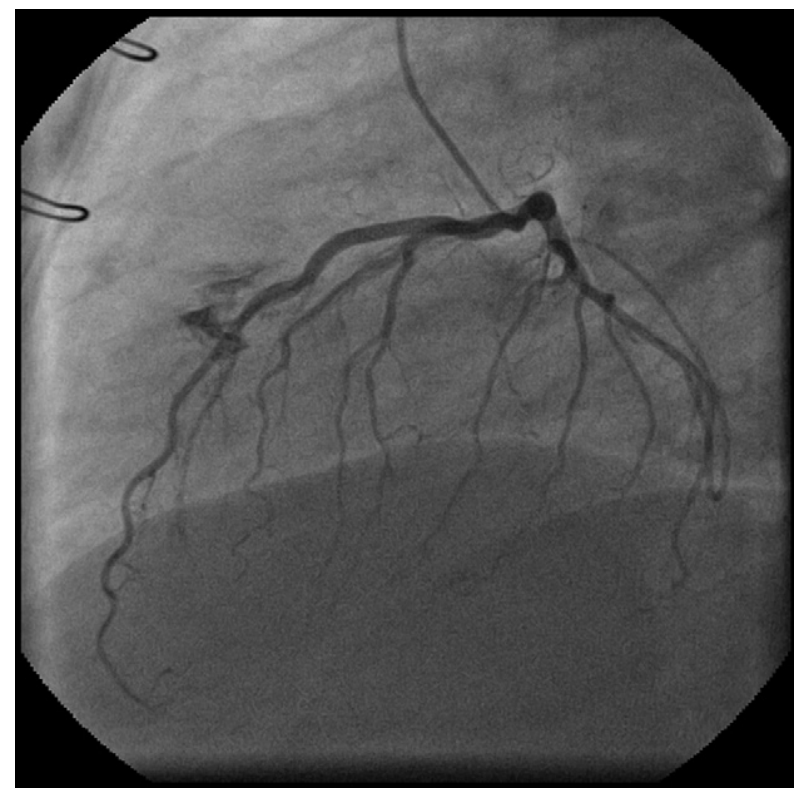

Fig 1. Fistula between the anterior descending artery and the right ventricle.

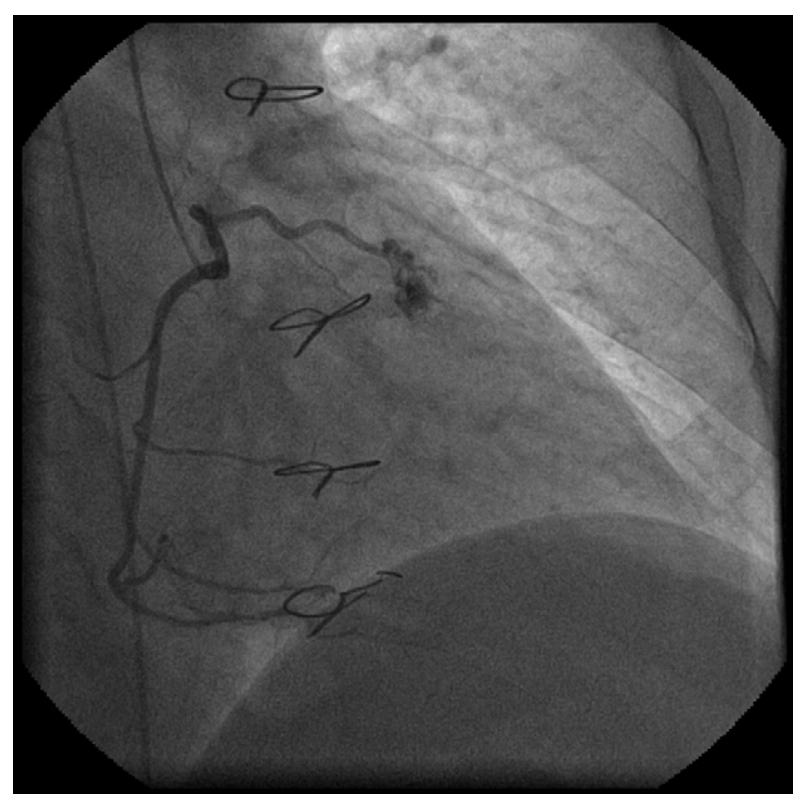

Fig 2. Fistula originating from the right ventricular branch of the right coronary artery and draining into the right ventricle.

ing walking and requires the patient to remain in bed for 2 hours postprocedure.

The average number of fragments collected per procedure was less than that described in other published studies, ${ }^{7,9,10}$ but the percentage of inappropriate specimens was clearly lower (4\% vs $9 \%$ ). ${ }^{7}$ In fact, sampling error is a real problem associated with EMB, which may result in a missed diagnosis or an underestimate of the severity of allograft rejection. In this study, only 12 EMB procedures were considered inconclusive. Despite collecting fewer specimens than usual, almost all the EMB procedures allowed a diagnosis of the absence or presence of rejection, and among the latter instances, a quantification of its severity. Therefore, despite the search for new noninvasive biomarkers, EMB remains the gold standard to monitor rejection in cardiac transplant patients, with a diagnostic rate previously reported to approach $100 \% .^{11,12}$

In this work, we have reported a low cumulative complication rate $(0.71 \%)$. Deckers et al, in a prospective study of 546 EMB performed between 1982 and 1989, reported a $6 \%$ overall complication rate. ${ }^{10}$ In that series, the worse result were probably due to the serious clinical state of the patients, characterized by severe left ventricular dysfunction and increased filling pressures. The high average number of collected specimens from each procedure $(6 \pm 2$ fragments) may also have contributed to the greater rate of problems. Other series published in the 80 s by the Mayo Clinic and by the Hosenpud group also demonstrated higher global complication rates: $4.4 \%$ and $14 \%$, respectively. $^{13,14}$

The preferential use of the femoral approach, the restriction of the EMB to the interventricular septum, the reduced number of collected fragments, the macroscopic analysis of 
all the fragments before sending them to the pathology laboratory, and the large experience of our center may have been responsible for our low complication rate. Only one patient had a major complication associated with the EMB: cardiac tamponade due to iatrogenic perforation of the right ventricle $(0.05 \%)$. Felker and colleagues described a perforation rate of $0.31 \%$ among 323 prospectively studied EMB. ${ }^{15}$ Frustaci and colleagues reported four cardiac perforations $(0.27 \%)$ among 1481 procedures. ${ }^{16}$

As in the study by Deckers et $a{ }^{10}{ }^{10}$ supraventricular arrhythmias were the most common tachyarrhythmias. This global arrhythmia incidence was higher than our observations $(1.1 \%$ vs $0.2 \%)$. As previously described in the literature, we also documented that patients with previous left bundle branch block showed a higher risk of developing complete AV block. ${ }^{17}$ The pressure exerted by the sheath or the bioptome on the right IVS can damage the right branch of the His bundle, producing complete block in patients with prior left bundle branch block. Therefore, continuous monitoring of these patients is always recommended.

$\mathrm{TR}$, the most common valvular abnormality in cardiac transplant patients, shows a prevalence ranging from $25 \%$ to $45 \% .^{18,19}$ Its etiology is mulfactorial. We have encountered valve tissue in nearly $11 \%$ of our patients. Mielniczuk and colleagues reported a similar rate of valvular fragment collection (9.2\%) among $205 \mathrm{EMB} .{ }^{20}$ Fiorelli et al reported a much lower rate $(3.2 \%),{ }^{21}$ probably due to the use of transthoracic echocardiography guidance during the EMB procedures. EMB may have a direct impact on the development of TR; however, the strength of this association remains controversial. In our series, as had also been shown by Fiorelli et $\mathrm{al}^{21}$ there was no significant correlation between accidental chordal tissue biopsy and the development of serious TR.

The incidence of CAF among our population was higher than that observed in the general population $(2.8 \%$ vs $0.1 \%-0.2 \%$ ), but lower than that previously shown in heart transplant patients $(5 \%-14 \%) .^{9,22,23}$ The etiology of CAF in heart transplant patients remains unclear. Some workers consider that trauma produced by repeat EMBs is the cause for the development of CAF., ${ }^{9,224}$ The presence of dilated arterioles in the histological specimens of transplanted patients with CAF has confirmed this association. However, a previous study found no difference in the number of EMB procedures in transplant patients with versus without CAF. ${ }^{25}$ Furthermore, in our population and also in another large study, ${ }^{9}$ patients who developed CAF did not show dilated arterioles upon histological examinations. As previously described, the majority of CAF showed a benign evolution. $^{26,27}$ The spontaneous closure of a fistula, observed in one of our cases, may have been due to the progression of the allograft coronary artery disease.

The several limitations of our study include its retrospective nature and the data being from a single heart transplantation center. Transthoracic echocardiography was not performed immediately before EMB, hence, we could not evaluate the development of small pericardial effusions as a direct consequence of this procedure.

In conclusion, EMB proved to show high diagnostic accuracy to identify graft rejection among heart transplant patients. The benefits derived from this invasive procedure were clearly superior to the risks. Serious complications were extremely rare. EMB can be performed with little or no discomfort; it is a safe procedure.

\section{ACKNOWLEDGMENTS}

We wish to thank to Marta Costa for all help provided during data collection.

\section{REFERENCES}

1. Yvengar S, Abraham WT: Anemia in chronic heart failure: can Epo reduce deaths? Cleve Clin J Med 72:1027, 2005

2. Edwards B, Daly R: Cardiac transplantation (100). In Murphy J, Lloyd M (eds): Mayo Clinic Cardiology Concise Textbook. Minnesota: Rochester; 2007, p 1179

3. Sutton D, Sutton Q, Kent G: Needle biopsy of human ventricular myocardium. Q Bull Northwest Univ Med Sch 30:213, 1956

4. Sakakibara S, Konno S: Endomyocardial biopsy. Jpn Heart J 3:537, 1962

5. Caves P, Schulz W, Dong E, et al: New instrument for transvenous cardiac biopsy. Am J Cardiol 33:264, 1974

6. Anderson J, Marshall H, Allison S: The femoral venous approach to endomyocardial biopsy: comparison with internal jugular and transarterial approaches. Am J Cardiol 53:833, 1984

7. Luis-Miranda R, Lázaro-Castillo J, Munayer-Calderón J, et al: Biopsia endiomiocardica. Revisión y experiencia de 176 procedimientos. Arch Cardiol Mex 77:200, 2007

8. Murphy J, Frantz R, Cooper L: Endomyocardial biopsy (123). In Murphy J, Lloyd M (eds): Mayo Clinic Cardiology Concise Textbook. Minnesota: Rochester; 2007, p 1481

9. Sandhu JS, Uretsky BF, Zerbe TR, et al: Coronary artery fistula in the heart transplant patient-a potential complication of endomyocardial biopsy. Circulation 79:350:6, 1989

10. Deckers JW, Hare JM, Baughman KL: Complications of transvenous right ventricular endomyocardial biopsy in adult patients with cardiomyopathy: a seven-year survey of 546 consecutive diagnostic procedures in a tertiary referral center. J Am Coll Cardiol 19:43, 1992

11. Fenoglio J, Ursell P, Kellogg C, et al: Diagnosis and classification of myocarditis by endomyocardial biopsy. N Engl J Med 308:12, 1983

12. Parillo JE, Aretz HT, Palacios I, et al: The results of transvenous endomyocardial biopsy can frequently be used to diagnose myocardial diseases in patients with idiopathic heart failure. Endomyocardial biopsies in 100 consecutive patients revealed a substantial incidence of myocarditis. Circulation 69:93, 1984

13. Nippoldt TB, Edwards WE, Holmes DR, et al: Right ventricular endomyocardial biopsy; clinicopathologic correlates in 100 consecutive patients. Mayo Clinic Proc 57:407, 1982

14. Hosenpud JD: Complications of endomyocardial biopsy. In Karson J, Morton MS (eds): Complications of Cardiac Catheterization and Angiography: Prevention and Management. Mt Kisco: Futura; 1989, p 135

15. Felker GM, Hu W, Hare JM, et al: The spectrum of dilated cardiomyopathy: the Johns Hopkins experience with 1278 patients. Medicine (Baltimore) 78:270, 1999

16. Frustaci A, Pieroni M, Chimenti C: The role of endomyocardial biopsy in the diagnosis of cardiomyopathies. Ital Heart $\mathbf{J}$ 3:348, 2002

17. Holzmann M, Nicko A, Kühl U, et al: Complication rate of right ventricular endomyocardial biopsy via the femoral approach: 
a retrospective and prospective study analyzing 3048 diagnostic procedures over 11 year period. Circulation 118:1722, 2008

18. Nguyen V, Cantarovich M, Cecere R, et al: Tricuspid regurgitation after cardiac transplantation: how many biopsies are too many? J Heart Lung Transplant 24:1586, 2005

19. Jeevanandam V, Russel H, Mather P, et al: Donor tricuspid annuloplasty during orthotopic heart transplantation: long term results of a prospective controlled study. Ann Thorac Surg 82:2089, 2006

20. Mielniczuk L, Haddad H, Davies RA, et al: Tricuspid valve chordal tissue in endomyocardial biopsy specimens of patients with significant tricuspid regurgitation. J Heart Lung Transplant 24: 1586, 2005

21. Fiorelli AI, Coelho GH, Oliveira JL, et al: Endomyocardial biopsy as risk factor in the development of tricuspid insufficiency after heart transplantation. Transplant Proc 41:935, 2009
22. Henzlova M, Nath H, Bucy RP, et al: Coronary artery to right ventricular fistula in heart transplant recipients: a complication of endomyocardial biopsy. J Am Coll Cardiol 14:258, 1989

23. Somers JM, Verney GI: Coronary cameral fistulae following heart transplantation. Clin Radiol 44:419, 1991

24. Sutsch G, Heywood T, Turina J: Coronary artery-right ventricular fistula in a heart transplant patient. J Heart Transplant 9:32, 1990

25. Lazar J, Uretsky B: Coronary artery fistula heart transplantation: a disappearing entity? Cathet Cardiovasc Diagn 37:10, 1996

26. Mahoney LT, Schieken RM, Lacer RM: Spontaneous closure of a coronary artery fistula in childhood. Pediatr Cardiol 2:311, 1982

27. Griffiths SP, Ellis K, Hordof AJ, et al: Spontaneous closure of a congenital coronary artery fistula. J Am Coll Cardiol 2:1169, 1983 